\title{
REVISIÓN DE CONCEPTOS FUNDAMENTALES DE LA PRÁCTICA BA- SADA EN LA EVIDENCIA
}

\author{
KEYS CONCEPTS REVIEW ON EVIDENCE BASED PRACTICE
}

\author{
Mónica Pérez S.* \\ Yolanda Contreras G. ${ }^{* *}$ \\ Sonia Olavaria B. ${ }^{* *}$
}

\begin{abstract}
RESUMEN
El objetivo de este artículo es dar a conocer conceptos fundamentales de la práctica basada en la evidencia para incentivar su aplicación en la atención clínica. El momento de una decisión clínica resulta particularmente complejo y relevante pues en él se conjugan los conocimientos y experiencia del profesional, el entorno y las circunstancias en las que se vive el proceso de salud-enfermedad, los valores y preferencias del afectado y su familia que serán sujeto de la decisión. La medicina basada en la evidencia es una herramienta que surge frente a la necesidad de aplicar las prácticas clínicas con eficiencia, eficacia y efectividad. Instituciones de conocido prestigio han desarrollado diversas orientaciones para la clasificación de las evidencias científicas disponibles, de tal manera que se constituyen en una guía para la práctica médica diaria en el momento de revisar artículos científicos de interés al caso particular que el profesional tenga que resolver.
\end{abstract}

Palabras clave: Medicina basada en evidencia, Práctica basada en la prueba.

\begin{abstract}
The purpose of this particular paper is to present keys concepts of evidence based practice to encourage its application in clinical attention. The moment when a clinical decision is made by a health professional is particularly critical and relevant because when this happens all of the knowledge, experience and the environment and circumstances of the health-illness process are connected and involved. Values and preferences of the patient and his family should also be considered and assessed. The generation of evidence based medicine is a new tool to be employed when the need to apply efficient and effective clinical practice is critical. Prestigious health centers and institutions have already developed new guidelines for the classification of available scientific evidence. Thus, they can guide daily medical practitioners when revision of pertinent scientific paper is necessary for specific cases to be solved.
\end{abstract}

Key words: Evidence Based Medicine, Evidence Based Practice.

Fecha recepción: 04/09/08 Fecha aceptación: 06/07/09

\footnotetext{
${ }^{*}$ Matrona. Magíster en Salud Familiar. Diplomada en Sexualidad Humana y Responsabilidad Social. Profesor asociado. Email: moperez@udec.cl

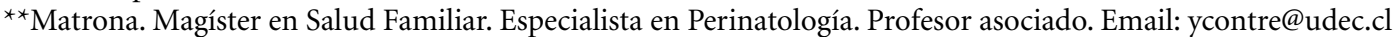

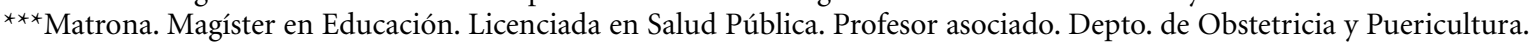
Facultad de Medicina. Universidad de Concepción. Chile. Email: solavarr@udec.cl
} 


\section{INTRODUCCIÓN}

El escenario en salud valoriza elementos asociados a la tecnología, los costos, el debate entre aspectos éticos - biológicos y la calidad de la atención.

Se ha observado la existencia de variaciones en la práctica diaria, comprobándose que algunas no están sustentadas por estudios científicos confiables. Frente a esta realidad, un grupo de médicos de la Universidad de Mc Master dio inicio a un nuevo enfoque en la enseñanza y práctica de la medicina, que denominaron Evidence Based Medicine (MBE), este término en español no tiene traducción literal por lo que la mejor traducción sería la medicina basada en la prueba (1); sin embargo ha ocurrido una suerte de traducción considerada un anglicismo léxico típico, esto es palabras escritas iguales en dos lenguas pero con significado distinto, y que al interpretarlas en su conjunto se reconocen con la concepción de la lengua que la origina. Este concepto o idea no es nuevo, ya que surge ligado a la introducción de la estadística y al método epidemiológico en la práctica médica. Su implementación se produce en la década de los 60 en la Universidad de Mc Master en Ontario, Canadá. Es en este centro en donde se define un nuevo enfoque para la Docencia de la Práctica Clínica, proponiendo un cambio en el modelo o paradigma del aprendizaje y la práctica de la medicina $(2,3)$.

El impulso fundamental de la MBE fue dado por Archie Cochrane en la década de los 70, a quien se le considera pionero en las investigaciones de los servicios sanitarios. Su libro "Efectividad y Eficacia: Reflexiones aleatorias en los Servicios de Salud" (4), es considerado crucial para la filosofía sanitaria y su mensaje principal es que no se puede mejorar la eficiencia sin pasar por la eficacia y la efectividad. Todos estos términos se encuentran asociados a la evaluación de la práctica diaria, afirmando que "es necesario establecer un mecanismo que garantice que las revisiones sistemáticas sean realizadas, actualizadas y divulgadas adecuadamente" (5).

En los años ochenta, otras organizaciones científicas comenzaron a trabajar basándose en estos objetivos, siendo una de las primeras la Oxford Database of Perinatal Trials (6).

La medicina basada en la evidencia o en la prueba, es una forma de abordar los problemas clínicos, utilizando para solucionarlos aquellos resultados originados en la investigación científica. En palabras de D L Sackett, de Oxford (7), se refiere a ella como "El uso consciente, explícito y juicioso de la mejor evidencia actual pertinente al cuidado de pacientes individuales. La práctica de la Medicina Basada en la Evidencia significa la integración de la experiencia y habilidad clínica individual con la mejor evidencia clínica externa emanada de revisiones sistemáticas (8). Los buenos médicos usan tanto su experiencia clínica como la mejor evidencia externa, pero ninguna de ellas por sí sola es suficiente. Sin la experiencia clínica, el ejercicio médico corre el riesgo de ser tiranizado por la evidencia externa, por cuanto aun la mejor evidencia externa puede ser inaplicable $\mathrm{o}$ inapropiada ante un paciente individual. Y sin la evidencia externa actualizada, el médico puede volverse rápidamente obsoleto, en detrimento de los pacientes". De tal forma que la evidencia reduce el uso de la intuición, la experiencia clínica no sistemática y la fisiopatología racional como base suficiente para la toma de decisiones clínicas y pone énfasis en el examen de evidencias para la investigación clínica (9-11).

\section{¿CÓMO SE ENCUENTRAN CLASIFICADAS LAS EVIDENCIAS?}

Existen varias propuestas para la clasificación de las evidencias. Una de las más conocidas y tal vez la más sencilla es la de United States Preventive Task Force (USPSTF) $(12,14)$, que clasifica las evidencias en 3 niveles, como se muestra en la Tabla 1. 
Tabla 1: Jerarquía de los estudios por el tipo de diseño según US Preventive Task Force

\begin{tabular}{|c|l|}
\hline $\begin{array}{c}\text { Nivel de } \\
\text { evidencia }\end{array}$ & \multicolumn{1}{c|}{ Tipo de estudio } \\
\hline I & $\begin{array}{l}\text { Al menos un ensayo clínico controlado y aleatorizado diseñado en forma } \\
\text { apropiada }\end{array}$ \\
\hline II-1 & Ensayos clínicos controlados bien diseñados, pero no aleatorizados \\
\hline II-2 & $\begin{array}{l}\text { Estudios de cohorte o caso control bien diseñados, preferentemente multi- } \\
\text { céntricos. }\end{array}$ \\
\hline II-3 & $\begin{array}{l}\text { Múltiples series comparadas en el tiempo con o sin intervención, y resul- } \\
\text { tados sorprendentes en experiencias no controladas. }\end{array}$ \\
\hline III & $\begin{array}{l}\text { Opiniones basadas en experiencias clínicas, estudios descriptivos, observa- } \\
\text { ciones clínicas o informes de comités de expertos. }\end{array}$ \\
\hline
\end{tabular}

Otra clasificación es la del Centro de $\mathrm{Me}$ dicina Basada en la Evidencia de Oxford (CEBM) $(13,14)$, no sólo evalúa las intervenciones terapéuticas y preventivas, sino también aquéllas ligadas al diagnóstico, pronóstico, factores de riesgo y evaluación económica, lo que es esquematizado en la Tabla 2.

Tabla 2: Niveles de evidencia según el CEBM

\begin{tabular}{|c|c|}
\hline $\begin{array}{l}\text { Nivel de } \\
\text { evidencia }\end{array}$ & Tipo de estudio \\
\hline $1 \mathbf{a}$ & Revisión sistemática de ensayos clínicos aleatorizados, con homogeneidad. \\
\hline $1 \mathrm{~b}$ & Ensayo clínico aleatorizado con intervalo de confianza estrecho \\
\hline $1 \mathrm{c}$ & Práctica clínica ( todos o ninguno)* \\
\hline $2 \mathbf{a}$ & Revisión sistemática de estudios de cohortes, con homogeneidad \\
\hline $2 \mathrm{~b}$ & Estudios de cohorte o ensayo clínico de baja calidad \\
\hline $2 \mathrm{c}$ & “Outcomes research"**, Estudios ecológicos \\
\hline $3 \mathbf{a}$ & Revisión sistemática de estudio caso control con homogeneidad \\
\hline $3 \mathbf{b}$ & Estudio caso-control \\
\hline 4 & Serie de casos o estudios de cohortes y caso-control de baja calidad \\
\hline 5 & $\begin{array}{l}\text { Opinión de expertos sin valoración crítica explícita, "bench research" o } \\
\text { "first principles" } \star \star \star\end{array}$ \\
\hline
\end{tabular}

* Cuando todos los pacientes mueren antes de que el tratamiento esté disponible, y con él algunos sobreviven, o bien cuando algunos pacientes morían antes de su disponibilidad y ahora no muere ninguno.

** "Outcomes research" se refiere a estudios de cohortes de pacientes con el mismo diagnóstico en los que se relacionan los eventos que suceden con las medidas terapéuticas que reciben.

*** Hace referencia a la adopción de determinada práctica clínica basada en principios fisiopatológicos. 
En general, los niveles propuestos se pueden explicar de la siguiente forma $(15,16)$ :

Nivel I: La evidencia es obtenida a partir de, al menos, un ensayo aleatorizado y controlado, diseñado de forma apropiada. Este tipo de investigación compara el efecto de un tratamiento con un placebo, administrados de forma tal que ni el que conduce el experimento ni el paciente conocen qué está recibiendo este último (doble ciego) y donde la asignación a cada grupo, tratamiento o control, se realiza en forma estrictamente aleatoria, evitando así los sesgos.

Aun así, existe la probabilidad de que el resultado favorable de un experimento sea el resultado del azar y no de la intervención. Para minimizar esto, un investigador define de antemano el tamaño de la muestra y cuál será la mayor probabilidad permitida al azar para cada uno de los desenlaces. Es lo que habitualmente se lee en los trabajos como una $\mathrm{p}=0.05$ que corresponde a un intervalo de confianza del $95 \%$, es decir que un $5 \%$ convencionalmente se está dejando al azar.

Si la muestra es de tamaño adecuado, la probabilidad de que el resultado se deba al azar se conoce como error alfa o tipo 1 y la probabilidad de no poder demostrar un efecto de la intervención cuando sí lo había, se conoce como error beta o tipo 2 .

Cuando se han desarrollado varios estudios clínicos controlados que sugieren la efectividad de un tratamiento pero carecen de poder para demostrarlo en forma contundente, se pueden reanalizar sus datos como si provinieran de un solo estudio. Así, aumenta el tamaño de la muestra y mejora considerablemente su poder. Para que los estudios puedan agruparse de esta forma, se requiere que cumplan ciertos requisitos que se refieren a la homogeneidad y a otros aspectos. El resultado es un metanálisis, que si se realiza correctamente, también puede considerarse como una evidencia del nivel I.

Nivel II: En este nivel la evidencia es obtenida de ensayos clínicos controlados sin aleatorización, que no alcanza un poder suficiente para probar en forma inequívoca la efectividad de una intervención, porque sus resultados se ubican alrededor del límite estadísticamente significativo. Al no existir aleatorización de los grupos en comparación pueden ser diferentes en sus variables pronósticas y afectar el resultado. Este nivel de evidencia también se puede obtener de estudios de cohorte o caso-control bien diseñados, realizados preferentemente en más de un centro o por un grupo de investigación, o a partir de múltiples series comparadas en el tiempo con intervención o sin ella. Estos tipos de estudios pueden ser considerados evidencia de nivel II siempre y cuando se someta a la aprobación de un grupo de consenso. En el mismo nivel se sitúa un informe de un comité de expertos (position statement), si presenta explícitamente su metodología.

Nivel III: Las evidencias están basadas en estudios de cohorte o ensayos clínicos controlados donde la aleatorización es deficiente o no se cumple, razón por la cual, se pueden introducir sesgos en la asignación de los pacientes a uno de los grupos del estudio (tratamiento o placebo). De igual forma ocurre cuando se compara la efectividad de tratamientos que se han establecido libremente en grupos de pacientes y cuya respuesta se observa al cabo de un determinado tiempo, estudio denominado de cohorte.

En ambas situaciones puede ocurrir, por ejemplo, que los casos más graves reciban el tratamiento A y no el B. Ante esta posibilidad, las evidencias de nivel III deben analizarse con cautela y, en la medida de lo posible, deben someterse a la aprobación de grupos de consenso.

Nivel IV: Comprende series de antes-después, estudios con cohorte histórica y estudios de caso-control. En esta situación, el resultado puede deberse a la acción de factores diferentes al tratamiento y que se hubieran podido descartar al incluir un grupo control bajo la influencia de los mismos factores.

También abarca aquellos estudios observacionales donde se compara el resultado de un nuevo tratamiento en una cohorte actual 
con los del tratamiento convencional que se utilizaba en el pasado (cohorte histórica) o se compara el tratamiento que recibieron los sujetos separándolos en dos grupos según la presencia o ausencia del desenlace (estudio de casos y controles).

En todos estos estudios pueden existir múltiples sesgos, razón por la que las evidencias de nivel IV deben analizarse con cautela y de la misma forma que la recomendación del nivel anterior, en la medida de lo posible debe someterse a la aprobación de grupos de consenso.

Otros estudios como las series de casos, informes de casos, opiniones de expertos, pueden ser útiles pero su nivel de evidencia es bajo. Sin embargo, en ocasiones pueden ser la única información disponible como ocurre, por ejemplo, con casos raros o con efectos secundarios inesperados.

Generalmente frente a la práctica clínica de la $\mathrm{MBE}$, las publicaciones se basan en revisiones sistemáticas. Esta se refiere a la búsqueda y evaluación crítica de todos los estudios primarios que dan respuesta a la misma pregunta. Es una revisión exhaustiva de la literatura acerca de una interrogante clara, utilizando una metodología sistemática y explícita para identificar, seleccionar y evaluar críticamente las investigaciones relevantes y para recolectar y analizar los datos provenientes de los estudios incluidos en la misma.

Para ello, una revisión sistemática debe ${ }^{1}$ :

1. Tener objetivos claros y establecidos, es decir la pregunta clínica que se desea responder esté claramente especificada en cuanto a: población de interés, intervención o exposición que se desea evaluar, y resultados que se desean medir.

2. La estrategia de búsqueda de la evidencia debe estar documentada y ser comprensible, para lo cual es necesario especificar los criterios de selección de las investigaciones que se incluirán en la revisión, en cuanto al tipo de pacientes (población), tipo de intervención, resultados a medir, y el tipo de diseño metodológico de las investigaciones que se incluirán.

3. La evidencia obtenida es sometida a una evaluación de calidad mediante un sistema explícito, en cuanto a su diseño, implementación y análisis para determinar si sus resultados son suficientemente confiables. La evaluación de calidad de los estudios debería ser realizada por más de una persona utilizando criterios preestablecidos, debería realizarse en forma independiente y en lo posible con el desconocimiento de los nombres de los autores y las revistas, los países de procedencia y los resultados, ya que estos datos podrían influir en la evaluación de calidad de los estudios e introducir sesgos.

4. La forma de combinar la evidencia de los estudios individuales debe estar claramente especificada. A veces los estudios de una revisión muestran resultados diferentes. Estas diferencias pueden deberse a que los pacientes son distintos, diferencias en el tratamiento administrado, diferentes formas de medir los resultados, diferente metodología de estudio, o por azar. Mediante una prueba estadística (test de homogeneidad) se puede evaluar la probabilidad de que las diferencias entre los resultados se deban al azar.

5. Cada revisión trabaja en base a los ensayos controlados y aleatorizados y sus comparaciones son presentadas en tablas y los resultados expresados como riesgo relativo (RR), con su correspondiente intervalo de confianza de $95 \%$ o $99 \%$.

El riesgo relativo permite:

- Reconocer la naturaleza como factor de riesgo o de protección de una exposición.

${ }^{1}$ Centro Latinoamericano de Perinatología y Desarrollo humano (CLAP) y Centro Rosarino de Estudios Perinatales. Medicina Basada en la Evidencia con énfasis en Perinatología. [Apuntes Taller]. 2001. Universidad de Concepción. Chile. 
- Identificar la magnitud o fuerza de la asociación, lo que permite hacer comparaciones.

En base a estas revisiones se han categorizado las prácticas de la siguiente forma (17):

1. Prácticas beneficiosas: A aquellos cuidados o tratamientos cuya efectividad está demostrada mediante una evidencia clara proveniente de ensayos clínicos controlados, siendo pequeñas las expectativas de posibles daños comparados con los beneficios.

\section{Prácticas probablemente beneficiosas:} Intervenciones cuya efectividad está menos establecida que para aquéllas clasificadas como "beneficiosas".

3. Prácticas de efectividad desconocida: Intervenciones para las que existen insuficientes datos; o bien, éstos son de calidad inadecuada.

\section{Prácticas probablemente no beneficiosas:} Intervenciones para las que la falta de efectividad está menos establecida que para las clasificadas como "perjudiciales o inefectivas".

5. Prácticas no beneficiosas: Intervenciones cuya inefectividad o perjuicio ha sido demostrada mediante una evidencia clara de estudios aleatorizados.

Actualmente, pocos tratamientos y prácticas clínicas son $100 \%$ seguras, beneficiosas o eficaces. La mayoría cae en un área grisácea, donde algunos pacientes se benefician y otros se perjudican. La práctica, basada en la evidencia, intenta controlar en algo esta incertidumbre, por lo cual su utilización es una nueva competencia que debe estar presente en los profesionales de salud (18).

Algunas barreras identificadas para la práctica de la $\mathrm{MBE}$ se refieren a: el estudio o la consulta en libros o textos obsoletos, publicaciones de investigaciones o revisiones sesgadas, la enorme cantidad de material científico publicado, para estar al día se requiere leer alrededor de 20 artículos diarios, dificultad para leer un artículo científico en forma crítica o para identificar si la literatura científica publicada es un estudio que tenga evidencia útil, capacidad para trasladar los resultados de las investigaciones a pacientes concretos y falta de tiempo (19).

Frente a estas barreras, algunas de las soluciones propuestas son: no leer libros como guías terapéuticas, no leer editoriales o estudios como guía si no son sistemáticas, mejorar sus habilidades para realizar una lectura crítica, leer primero revisiones de buena calidad y luego ir a los artículos originales que efectivamente sean un aporte, aprender a manejar software bibliográfico, mejorar el conocimiento de las interpretaciones estadísticas ${ }^{2}$.

El aporte de la MBE para la práctica clínica no sólo se refiere a las efectividad de la práctica sino que se pueden identificar claramente aportes para los profesionales de salud como para los usuarios de los sistemas sanitarios $(20,21)$.

Ventajas para los profesionales:

- Actualizar sus conocimientos de forma rutinaria.

- Mejorar su comprensión acerca de los métodos científicos y ser más críticos cuando utilicen datos.

- Incrementar su confianza en las decisiones relacionadas con la gestión.

- Aumentar su capacidad para utilizar las fuentes de información bibliográficas y sus hábitos de lectura.

- Reforzar la cohesión de los equipos clínicos al establecer un marco objetivo de funcionamiento. 
Ventajas para los usuarios:

- Disminución en la variabilidad de prácticas y acceso a prestaciones más eficaces.

- Permite una comunicación adicional y operativa con proveedores de atención en salud, pues se objetiviza los pro y contra de cada opción terapéutica.

- Participación real en la toma de decisiones que les afectan.

Si la información que se maneja es suficientemente ágil y apropiada, puede ser comprendida perfectamente por numerosos usuarios y grupos de consumidores, con lo que se contribuye también a la democratización real del conocimiento (20).

\section{CONSIDERACIONES FINALES}

De acuerdo a lo presentado, la práctica clínica se plantea hoy como una conjunción que involucra la investigación rigurosa, diseñada y ejecutada, y no sólo la autoridad de la experiencia clínica, de lo anecdótico y de lo empírico. Es decir, valorando lo que se dice, cómo se dice y quién lo dice, apoyado siempre en la mejor evidencia (22).

En definitiva, lo que pretende la $\mathrm{MBE}$ es que esta práctica se adecue a la investigación clínica disponible de modo que, una vez localizada y evaluada por los profesionales de salud, sea aplicada para mejorar el cuidado de sus pacientes y su propia práctica $(23,24)$.

La vertiginosidad del avance científico obliga a los profesionales de salud a revisar en forma permanente la mejor evidencia clínica disponible. La MBE es un aporte que debe tenerse en consideración por cualquier profesional de salud que realice práctica clínica (21).

\section{REFERENCIAS}

1. Muñoz F, Cabrera F. Reflexiones sobre la evidencia en medicina. Revista Médica de Chile. 2005; 133 (10):1252-1257.

2. Guerra L. La Medicina Basada en la Evidencia: un intento de acercar la ciencia al arte de la práctica clínica. Medicina Clínica Barcelona. 1996; 107:377-82.

3. Letelier LM, Moore Ph. La Medicina Basada en la Evidencia. Visión después de una década. Revista Médica de Chile. 2003; 131 (8): 939-946.

4. Cochrane Collaboration. [citado 2008 enero 10]; Disponible en: http://www. cochrane.org/

5. Patiño J. Medicina Basada en la Evidencia. Medicina Bogotá. 1999; 21(50):5-14. [citado 2007 diciembre 18]; Disponible en http://www.encolombia.com/medicina/ academedicina/j-03mbe.htm

6. Curley AE, Halliday HL. Pediatría basada en la evidencia. Anales de Pediatría, 2000; 52 (06): 497-500.

7. Sackett DL. Evidence Based Medicine: what it is and what it isn't. British Medical Journal. 1996; 312:71-72. [citado 2007 diciembre 12]; Disponible en: http://www. svnp.es/Documen/mbe.pdf.

8. González de Dios J. Introducción a la medicina basada en la evidencia. Boletín de la Sociedad Pediátrica de Asturias, Cantabria Castilla y León. 2001: 41; 201-244.

9. Díaz J, Gallego B. Medicina Basada en Evidencias: Controversias actuales. Revista Cubana Medicina General Integral. 2004; 20(3). [citado 2007 diciembre 12]; Disponible en: http://bvs.sld.cu/revistas/ mgi/vol20_3_04/mgi08304.htm

10. Marti A . La Medicina Basada en Evidencia en 1948: Visión desde 2005. Gaceta Médica de Caracas. Venezuela. 2006; 114 (3):208-213.

11. Patiño M. La Medicina Basada en la Evidencia como modelo de decisión clínica. Rev. Ven Urol 2005; 51(1): 20-29. 
12. United States Preventive Task Force (USPSTF). [citado 2007 diciembre 18]; Disponible en: http://www.ahrq.gov

13. Centro de Medicina Basada en la Evidencia de Oxford. [citado 2007 diciembre 18]; Disponible en: http://www.cebm.net/ levels_of_evidence.asp

14. Primo J. Niveles de Evidencia y Grados de Recomendación. Ponencia presentada en el Simposium "Gestión del conocimiento y su aplicación en la enfermedad inflamatoria crónica intestinal”. Valencia, España, 24 de enero de 2003. [citado 2007 diciembre 13]; Disponible en: http://www.svpd.org/ mbe/niveles-grados.pdf.

15. Broche JM, Broche RC, García L. Medicina Basada en la Evidencia: un reto para el médico contemporáneo. ACIMED. [online], 2003; 11 (6) [citado 2008 enero 10]; Disponible en: http://www.bvs.sld.cu/ revistas/aci/vol11_6_03/aci02603.htm

16. Hernández L. ¿Qué es la salud pública basada en la evidencia? Revista de Salud Pública Bogotá, Colombia. 2003: 5(1);4045: [citado 2008 enero 10]; Disponible en: www.opas.org.br/servico/arquivos/ sala566.pdf

17. Cochrane Clinical Collaboration. A guide to effective care in pregnancy and childbirth. 2001. [ citado 2007 diciembre 12]; Disponible en: http://www. cochranecollaboration.org

18. González de Dios J. La valoración crítica de documentos científicos y su aplicabilidad a la práctica clínica: aspecto clave en la toma de decisiones basada en las mejores pruebas científicas. Evidencias en Pediatría. 2006; 2. [citado 2007 diciembre 12]; Disponible en: http://www.aepap.org/EvidPediatr/ numeros/vol2/2006_numero_2/2006_ vol2_numero2.22.htm

19. Hinojosa M: Medicina Basada en la Evidencia: un nuevo reto al profesional de la información en salud. ACIMED. [online].2001: 9(1): 5-11. [citado 2008 enero 10]; Disponible en: http://bvs.sld. cu/revistas/aci/vol9_1_01/aci011001. htm.

20. Boucourt R, Larissa. Su Excelencia: la medicina basada en evidencias. ACIMED. 2003; 11 (3).

21. Rada G, Andrade M. ¿Debo aplicar los resultados de este estudio a mi paciente? Revista Médica de Chile, 2006; 134 (1):115-119. [citado 2008 marzo 4]; Disponible en: http://bvs.sld.cu/revistas/ aci/vol11_3_03/aci02303.htm

22. Moore Ph, Rivera S, Corbalán J. Cómo transmitir la evidencia a los pacientes: el médico como comunicador de riesgo. Revista Médica de Chile. 2006; 134 (3):385390.

23. Barroso M. Medicina Basada en Evidencias. Rev. Cubana de Oncología. 2000; 16 (2):135-36.

24. Orellana A, Paravic T. Enfermería basada en evidencia: barreras y estrategias para su implementación. Cienc. Enferm. 2007; 13(1): 17-24. [citado 2008 marzo 4]; Disponible en: http://www.scielo.cl/scielo. php?script $=$ sci_arttext\&pid $=$ S0717$95532007000100003 \& \operatorname{lng}=$ es. 